\title{
Nonsurgical Treatment of Hemifacial Microsomia: A Case Report
}

\author{
Mahtab Nouri and Arash Farzan ${ }^{2, *}$ \\ ${ }^{1}$ Orthodontic Department of School of Dentistry, Dentofacial Deformities Research Center, Research Institute of Dental Sciences, Shahid Beheshti University of Medical Sciences, \\ Tehran, IR Iran \\ ${ }_{2}^{2}$ Department of Orthodontics, School of Dentistry, Zanjan University of Medical Sciences, Zanjan, IR Iran \\ ${ }^{*}$ Corresponding Author: Arash Farzan, Department of Orthodontics, School of Dentistry, Zanjan University of Medical Sciences, Zanjan, IR Iran. Tel: +98-9126009396, Fax: +98- \\ 2122092644, E-mail:dr.arash.farzan@gmail.com \\ Received: May 13, 2014; Revised: June 29, 2014; Accepted: August 4, 2014
}

\begin{abstract}
Introduction:Hemifacial microsomia(HFM) is a birth defect involving craniofacial structures derived from the first and second branchial arches. Although it is a relatively uncommon malformation, it is the second most common craniofacial birth defect after cleft lip and palate $(\mathrm{CL} / \mathrm{P})$.

Case Presentation: This is a case report about the successful orthodontic treatment of a patient with mild hemifacial microsomia (HFM), using a non-surgical orthopedic and orthodontic treatment approach. The aim of this approach was to make the best noninvasive modality to treat HFM. A 7-year-old boy with a mild HFM presented with a convex profile and slight chin deviation. Orthopedic treatment performed using a hybrid functional and high pulls headgear. Treatment continued by fixed orthodontic straight wire appliance to achieve perfect occlusion.

Conclusions: Excellent esthetic and functional results achieved; total treatment duration was about 72 months.
\end{abstract}

Keywords: Orthodontics; Microsomias; Craniofacial; Functional Orthodontic Appliances

\section{Introduction}

Hemifacial microsomia (HFM) is a birth defect involving craniofacial structures derived from the first and second branchial arches and affects the orbit, maxilla, mandible, ear, cranial nerves and soft tissues, which usually results in a unilateral failure of formation primarily in the mandible and overlying structures. The malformation is also known in the literature as otomandibular dysostosis, Goldenhar syndrome, first and second branchial arch syndrome, lateral facial dysplasia and craniofacial microsomia (1).

Although HFM is a relatively uncommon malformation, it is the second most common craniofacial birth defect after cleft lip and palate $(\mathrm{CL} / \mathrm{P})$. The reported incidence varies widely but most frequently reported as 1:3000 to 1:5600 (2). Males are more affected than females with the estimate ratio of 3:2 and the right side of the face is affected more often than the left side with a ratio near 3:2 (3).

The etiology of HFM is not understood completely; however, it has been suggested to be in relation with a developmental abnormality mainly due to hemorrhage of the stapedial artery (4).

The treatment approaches for involved individual mainly related to the severity of the malformation. Several clinical classifications have been developed addressing the severity of HFM (1). These classifications help cli- nicians to select the best treatment amongst available treatment modalities. In mild and some moderately involved growing individuals, routine treatment involves a nonsurgical orthopedic treatment with hybrid functional appliance completed through fixed orthodontic treatment; however, more severely affected patients may need surgical intervention for distraction osteogenesis (DO), costochondral grafts or other hard/soft tissue surgeries. After pubertal growth, mild deformities can be corrected with orthodontic treatment alone or in conjunction with genioplasty and/or unilateral mandibular augmentation. However, more severe cases may require advanced interventions; for instance, simultaneous mandibular and maxillary surgery combined with hard/soft tissue augmentation procedures (5).

The purpose of this clinical case report was to represent a non-surgical approach using a hybrid functional appliance, headgear and fixed orthodontics to treat an involved adolescent boy with mild HFM.

\section{Case Presentation}

A 7-year-old Iranian boy was attended to private orthodontic office with mild HM. In clinical examination, an asymmetric face and slight chin deviation to left detected in addition to strain on lip closure on chin soft tissue. The maximum mouth opening was at normal range and both

Copyright ( 2015, Iranian Red Crescent Medical Journal. This is an open-access article distributed under the terms of the Creative Commons Attribution-NonCommercial 4.0 International License (http://creativecommons.org/licenses/by-nc/4.0/) which permits copy and redistribute the material just in noncommercial usages, provided the original work is properly cited. 
Nouri M et al.

condyles showed proper translatory movement; however, a deviation to left was present in opening. There was a maxillary cant of occlusion due to vertical deficiency of the mandible on the left side. There were no signs or symptoms of tempromandibular joint disease (TMD). The left ear was severely malformed and the left external auditory canal was absent. A reconstructive ear surgery was performed previously before beginning school at age of six years. The patient's profile was convex and a mild chin deficiency was present (Figure 1A - C).

Intra-oral examination revealed an increased overjet. The upper and lower incisors and first molars were completely erupted. The upper incisors were flared and spaced and a 2-mm midline deviation to right in comparison to facial midline was present in the upper arch. There was a $5.5 \mathrm{~mm}$ midline deviation between maxillary and mandibular midlines. In the upper and lower arches, the left first deciduous molars had a history of pulpotomy and large restorations accompanied with increased clinical mobility (Figure 1D - H).

The space analysis using dental casts and radiographic tooth size prediction revealed a $+2 \mathrm{~mm}$ tooth size-arch length discrepancy (TSALD) in the upper and +3 TSALD in the lower arch, respectively. The inter-arch molar relationship was CL II Angle on the right and CL III Angle on the left side, respectively.

Radiographic evaluation showed normal TMJ configuration including normal condyles on both sides. Cephalometric measurements revealed an asymmetric mandible with deficiency in sagittal plane. Both jaws showed a clockwise rotation and the gonial angle and sum of posterior angles were slightly increased, indicating a tendency toward vertical growth pattern (Figure 1I and H).

\subsection{Treatment Objectives}

Considering collected data on clinical examination and radiographic evaluation, the following objectives should be achieved:

1. Correcting the mandibular deviation and maxillary cant of occlusion

2. Correcting the mandibular deficiency

3. Obtaining vertical growth control preventing excessive mandibular clockwise rotation

4. Monitoring proper sequence of permanent teeth eruption and achieving an esthetic, functional and stable occlusion and facial appearance finally.

\subsection{Treatment Alternatives}

According to clinical and radiographic findings the treatment alternatives were:

1. An early surgical intervention for placing distractors and performing distraction osteogenesis (DO) for correcting vertical and sagittal mandibular deficiency on the left side, followed by comprehensive orthodontic treatment.

2. Two phase orthopedic/orthodontic treatment including growth modification and redirection by hybrid func- tional appliance and head gear (HG) followed by comprehensive fixed orthodontic treatment. Hybrid functional appliances constructed from acrylic and wire elements provide a proper environment for dentoalveolar and skeletal correction by forcing the mandible to be positioned correctly in the three dimensions of space. The resultant soft tissue stretch would help this phenomenon.

\subsection{Treatment Plan}

Considering the mild severity of the mandibular asymmetry and proper translatory movement of the both condyles in the glenoid foss, the DO approach discluded. Therefore, the second treatment alternative was selected for patient including growth modification followed by comprehensive fixed orthodontic treatment.

\subsection{Treatment Progress}

The right upper and lower first deciduous molars could not be restored, therefore extracted. Band and loop space maintainers were placed on the both right upper and lower second deciduous molars. Then an alginate impression was taken and wax construction bite was obtained to construct a hybrid functional appliance to advance mandible, bring the chin to the midline and correct vertical height deficiency in the left side. The hybrid functional appliance included a bite block on the right side and lingual and buccal shields on the left in addition to labial bows and retentive elements.

The functional treatment with hybrid appliance remained for 13 months with perfect compliance and nearly full-time wearing of the appliance. After this period, the mandibular deviation and vertical deficiency almost totally resolved and the treatment continued for another four months instructing the patient to wear appliance only at night during sleep (Figure $2 \mathrm{~A}-\mathrm{H}$ ).

After this short maintenance period, the functional hybrid appliance discontinued; upper first molars were banded and a high pull HG was applied for vertical control. The patient continued wearing HG for about 14 months until establishment of a full permanent dentition. Then a full bonded orthodontic edgewise appliance was placed (Roth 22) and the second phase of treatment initiated reserving the high pull HG wearing. After initial leveling and alignment by 0.0175 stainless steel (SS) arch wire, the conventional sequence of wires placed up to $0.018 \mathrm{SS}$ wire. CL II and CL III 3/16 medium elastics were applied on the left and right sides respectively for obtaining bilateral CL I dental relationship and preventing jaw deviation. 18.25 SS arch wires were used in finishing stage and final occlusion settling obtained on 16 SS arch wires using bilateral box elastics. Finally, occlusal adjustment was performed for achieving a proper functional and mutually protected occlusion. The second phase of treatment with fixed orthodontic appliance lasted for about 45 months. 

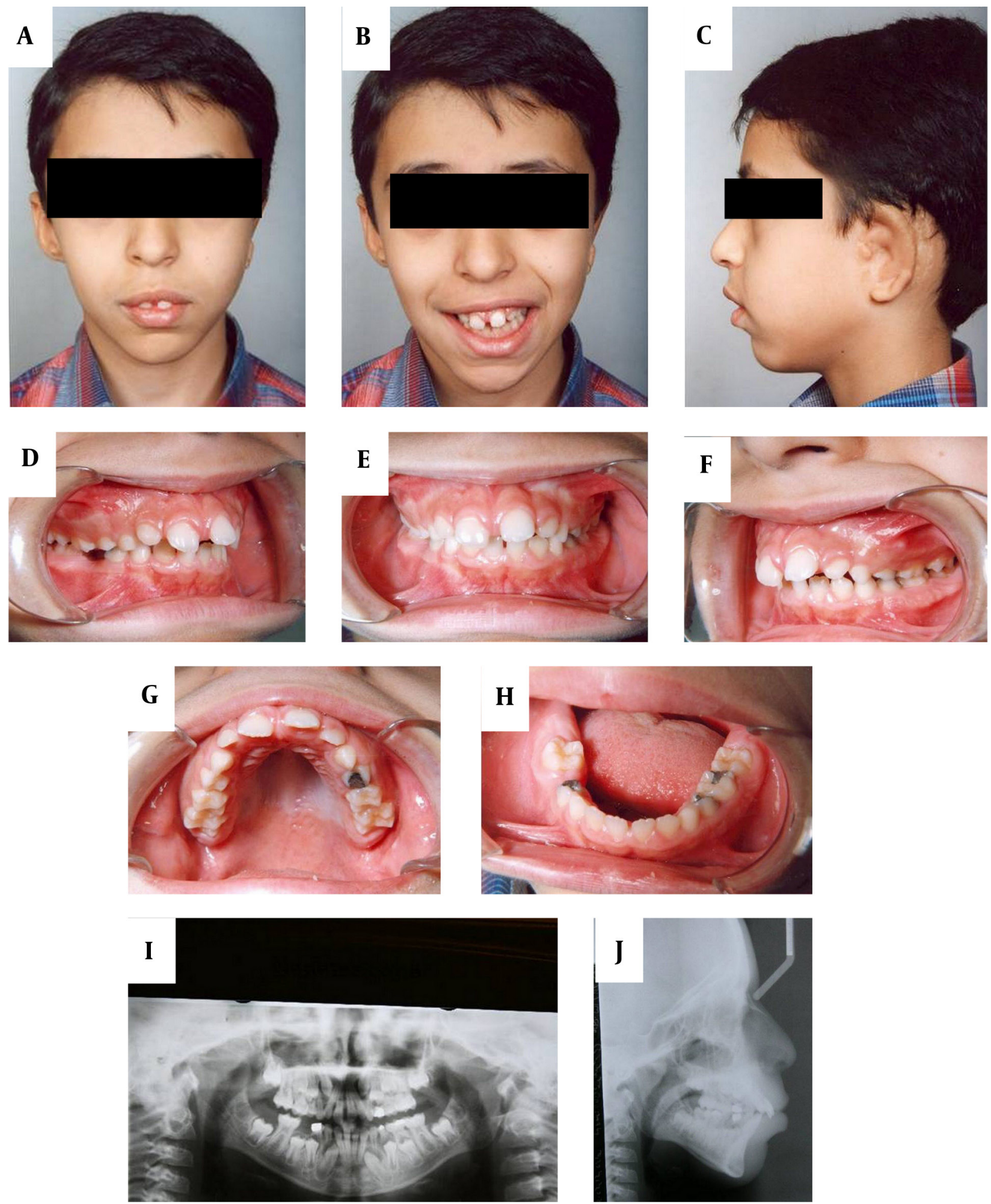

Figure 1. Extraoral and Intraoral Pretreatment Photographs, Panoramic View and Lateral Cephalogram. 

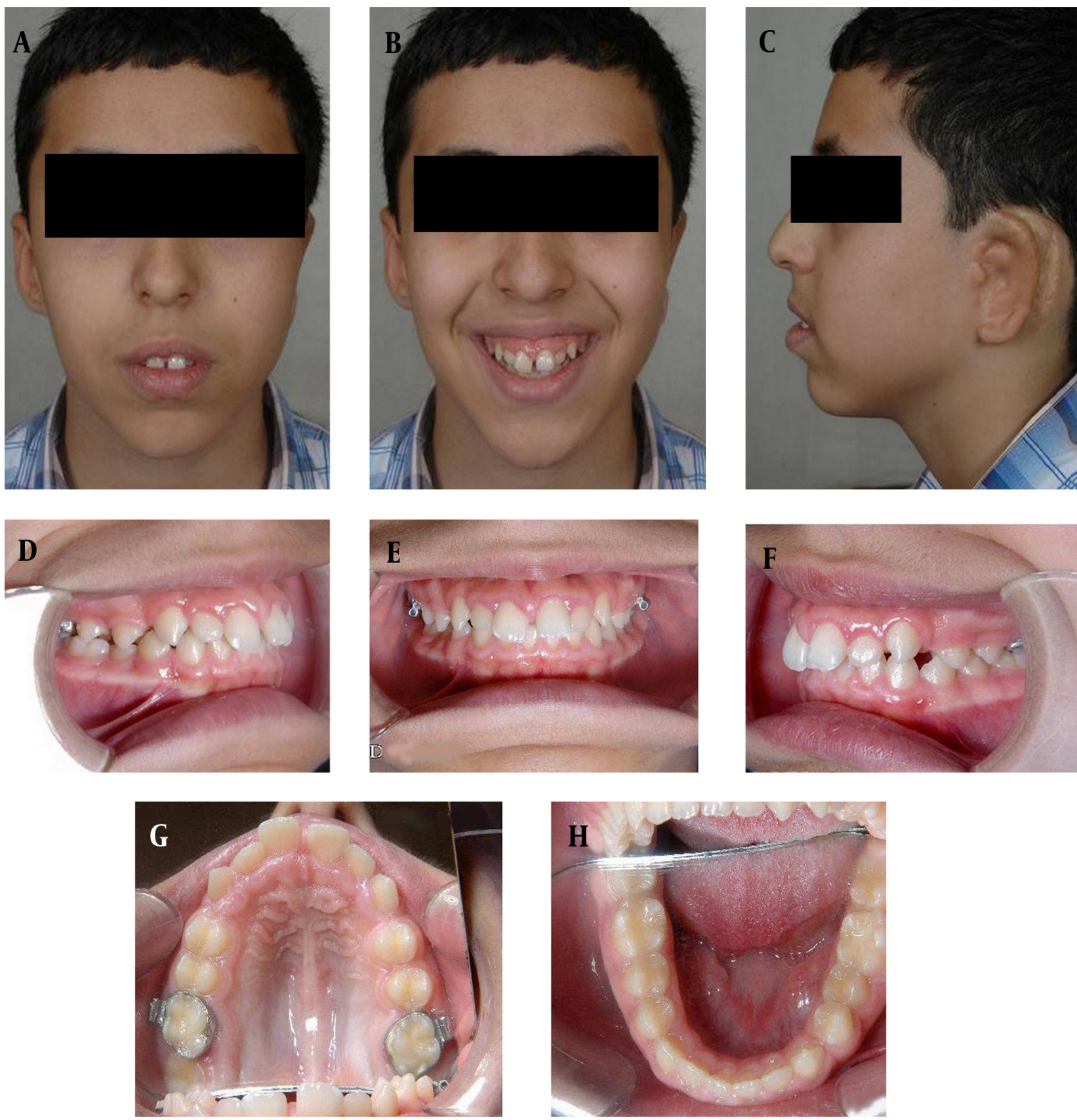

Figure 2. Extraoral and Intraoral Photographs After Phase I of Treatment.

Removable modified Hawley retainers with lingual shields for maintaining jaw relationship were used for retention. Patient was instructed to wear the retainers full-time, except at the meals and brushing for the first 12 months. Then the retainer wearing time reduced to nights only during sleeping.

\subsection{Treatment Results}

After completion of the first phase of treatment by means of hybrid functional appliance following high pull HG for vertical control, the facial asymmetry and mandibular deficiency were largely resolved. However, a fixed appliance 

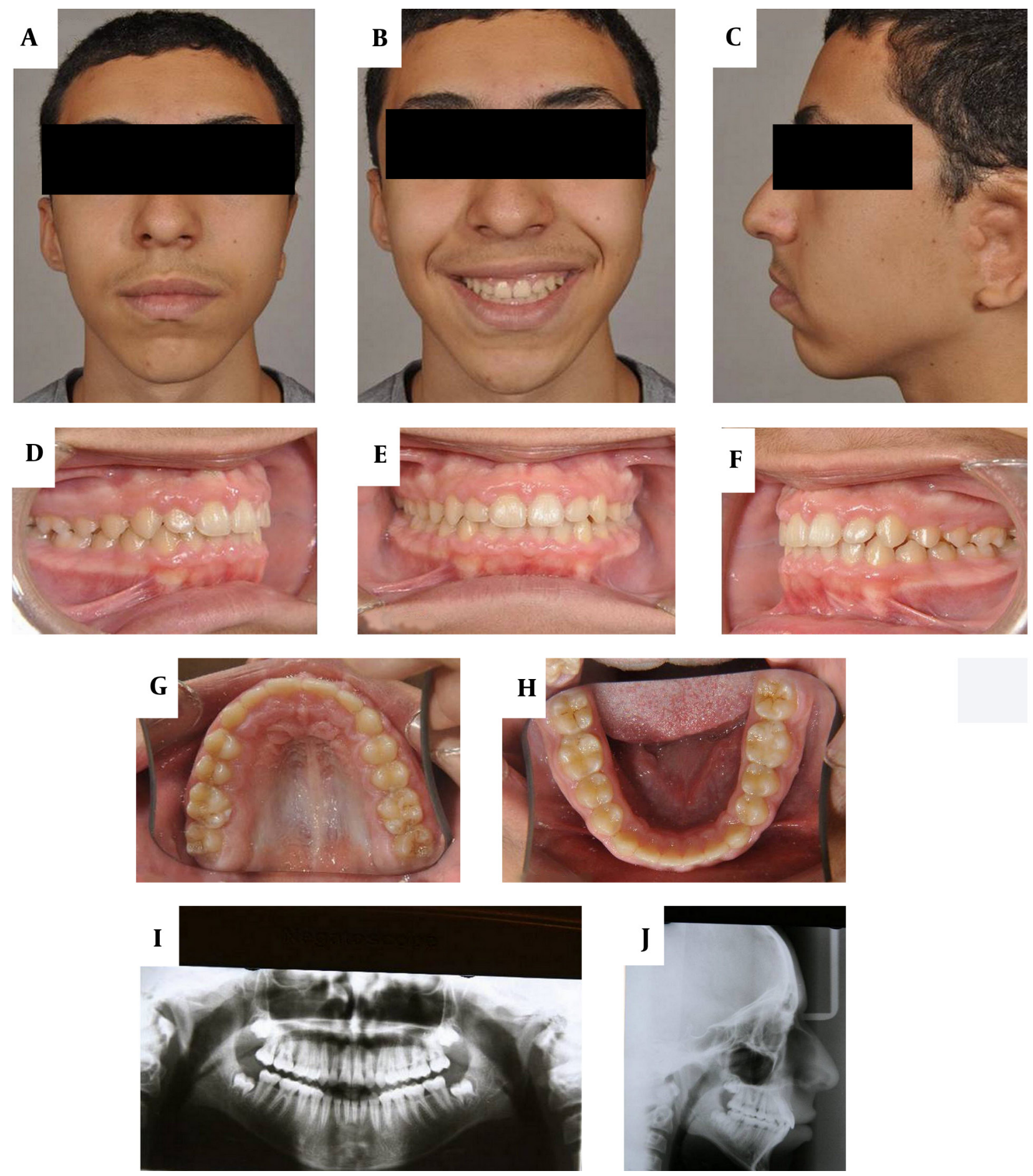

Figure 3. Extraoral and Intraoral Post-Treatment Photographs, Panoramic View and Lateral Cephalogram.

treatment was necessary to obtain optimum esthetic, functional and stable results. At the end of fixed orthodontic treatment, bilateral CL I occlusal relationship and normal overjet and overbite were achieved (Figure
$3 \mathrm{~A}$ - J). Total treatment time was about 72 months. The cephalometric tracing super impositions are presented in Figure 4 and before and after completed treatment measurements are presented in Table 1, respectively. 


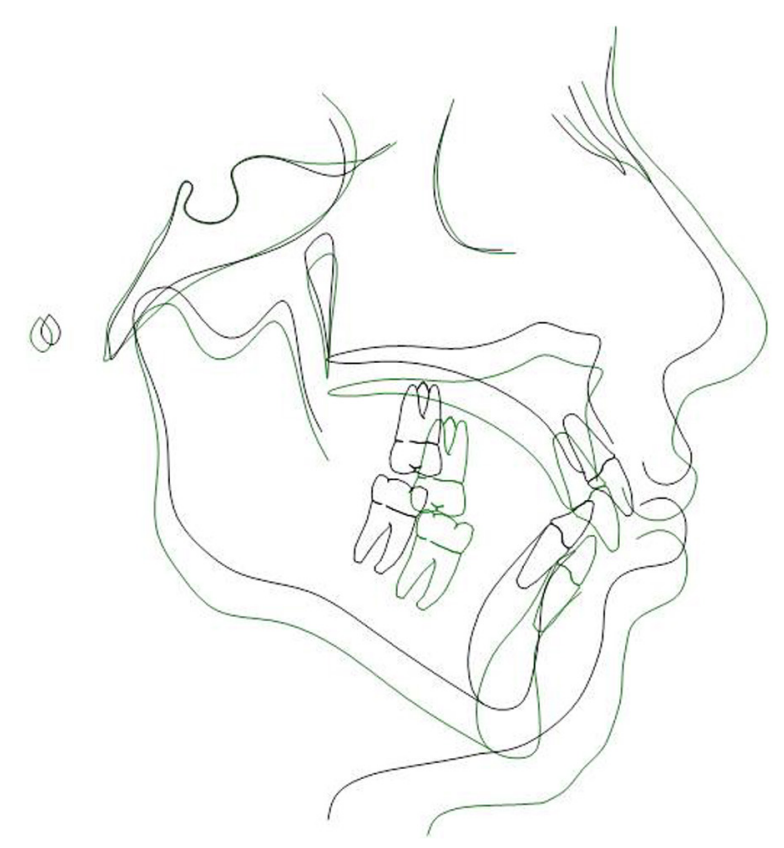

Figure 4. Pretreatment and Post-treatment Tracing Superimpositions

\begin{tabular}{lccc}
\hline \multicolumn{4}{l}{ Table 1. Analytical Measurements Before and After Treatment } \\
\hline Angle & $\begin{array}{c}\text { Value Before } \\
\text { Tx }\end{array}$ & $\begin{array}{c}\text { Value After } \\
\text { Tx }\end{array}$ & Norm \\
\hline SNA & 77 & 73.2 & 82 \\
\hline SNB & 69.5 & 67.3 & 80.9 \\
\hline ANB & 7.5 & 5.8 & 1.6 \\
U1-SN & 103.9 & 97.3 & 103.1 \\
\hline IMPA & 102.6 & 94 & 95 \\
\hline FMA (MP-FH) & 36.2 & 34.6 & 22.9 \\
\hline Gonial & 130.3 & 121.6 & 120.8 \\
\hline
\end{tabular}

${ }^{a}$ Abbreviations: A, Point A; B, Point B; Gonial, angle between mandibular plane and ramus plane; L-1, long axis of decidous mandibular central incisor; L-1 to mandibular plane, angle between L-1 and mandibular plane; Mandibular plane, angle between mandibular plane and FH plane; N, nasion; S, sella turcica; SNA, angle between SN and NA; SNB, angle between $\mathrm{SN}$ and NB; U-1, long axis of deciduous maxillary central incisor; U-1 to FH plane, angle between U-1 and FH (Frankfort horizontal) plane.

\section{Discussion}

Hemifacial microsomia represents a wide spectrum regarding the severity of facial structures involvements. In mildest representations, the affected side may only show decreased growth in all the three dimensions. However, in case of more severe involvement, showing deficient or absent condyles and improper mouth opening/TMJ func- tion, surgical intervention in the form of costochondral grafts or DO would be inevitable (5).

The orthodontist role is important in the treatment of these patients. In the mild forms of involvement such as the presented case here, proper orthopedic/orthodontic intervention can eliminate the need for surgery. The timing of treatment and patient compliance are very important in this regard (6). Fortunately, our patient was referred to our office at a proper age and strongly enthusiastic to follow clinician's instructions.

Following the first phase of treatment, almost always a second phase of orthodontic therapy by means of full fixed orthodontic appliance is required. This phase includes establishment of esthetic and functional occlusion and stabilizing the first phase results by dental compensation. This involves extrusion of buccal segment teeth on the affected side and resolving maxillary cant of occlusion (5). In this case, we also used a high pull HG to limit the vertical growth tendency. This strategy helped to prevent weak chin appearance due to clockwise rotation of the mandible as a consequence of vertical growth pattern.

For maintaining the results, long-term retention has been suggested (5). This involves fixed retainers and night time wearing of hybrid functional appliance. In this case, we used a modified Hawley with vertical lingual shield to prevent mandibular deviation to the affected side. In conclusion, the present case report indicated that mild forms of HM can be treated successfully by proper orthopedic and orthodontic intervention. This can eliminate further need to orthognathic surgery.

\section{Authors' Contributions}

Mahtab Nouri was the provider of presented case and instructor of preparing the manuscript. The manuscript was written by Arash Farzan and the final proof made by Mahtab Nouri.

\section{References}

1. Fan WS, Mulliken JB, Padwa BL. An association between hemifacial microsomia and facial clefting. J Oral Maxillofac Surg. 2005;63(3):330-4.

2. Grabb WC. The first and second branchial arch syndrome. Plast Reconstr Surg. 1965;36(5):485-508.

3. Rollnick BR, Kaye CI, Nagatoshi K, Hauck W, Martin AO. Oculoauriculovertebral dysplasia and variants: phenotypic characteristics of 294 patients. Am J Med Genet. 1987;26(2):361-75.

4. Cousley RR, Wilson DJ. Hemifacial microsomia: developmental consequence of perturbation of the auriculofacial cartilage model? Am J Med Genet. 1992;42(4):461-6.

5. Moulin-Romsee C, Verdonck A, Schoenaers J, Carels C. Treatment of hemifacial microsomia in a growing child: the importance of co-operation between the orthodontist and the maxillofacial surgeon. J Orthod. 2004;31(3):190-200.

6. Seow WK, Urban S, Vafaie N, Shusterman S. Morphometric analysis of the primary and permanent dentitions in hemifacial microsomia: a controlled study. J Dent Res. 1998;77(1):27-38. 